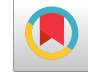

\title{
Primary Cardiac Tumors in Children: An 8-Year-Experience in a Single Center
}

\author{
Maryam Moradian ${ }^{1}$, Batool Hejazi Zadeh ${ }^{1}$, Fariba Rashidi Ghader ${ }^{1}$, Mohammad Rafie Khorgami ${ }^{1,}$, \\ Kambiz Mozaffari ${ }^{1}$, Fatemeh Mohammad Sadeghi ${ }^{2}$ and Hamidreza Pouraliakbar ${ }^{1}$ \\ ${ }^{1}$ Rajaie Cardiovascular Medical and Research Center, Iran University of Medical Sciences, Tehran, Iran \\ ${ }^{2}$ Iran University of Medical Sciences, Tehran, Iran \\ "Corresponding author: Rajaie Cardiovascular Medical and Research Center, Iran University of Medical Sciences, Tehran, Iran. Email: rafikhorgami@gmail.com
}

Received 2020 August 31; Revised 2020 October 06; Accepted 2020 October 19.

\begin{abstract}
Background: Cardiac neoplasms are not common in the pediatric population, and most of the reported cases in the literature are benign. Despite their benign nature, however, cardiac neoplasms may lead to hemodynamic instability and arrhythmias.

Objectives: Our study aimed to review the natural history, diagnosis, pathological features, and outcome of pediatric patients with cardiac tumors presenting to a tertiary pediatric cardiac center over an 8-year period.

Methods: This retrospective observational study reviewed and analyzed the hospital records and data of pediatric patients diagnosed with cardiac tumors over an 8-year period (2007-2015).

Results: During the period covered by the study (2007 - 2015), a total of 62975 pediatric in and out patients were referred to our tertiary cardiac center for cardiac evaluation. Cardiac tumors were identified in 41 patients, in 35 of whom tumor types were recognized. Rhabdomyomas were the most common tumors, followed by fibromas and myxomas. Fourteen patients underwent surgical management (either total or partial resection), and 4 patients received chemotherapy. Associated congenital heart diseases, except for the patent foramen ovale, were detected in12 patients.

Conclusions: While almost all our findings were compatible with previous data, we had a comparatively large number of undetermined tumors. Therefore, a better understanding of the imaging characteristics of cardiac tumors is needed for a better diagnosis of tumor types via noninvasive modalities.
\end{abstract}

Keywords: Cardiac Tumors, Primary Tumors, Rhabdomyoma, Fibroma, Children

\section{Background}

Cardiac neoplasms are rare in infants and children, and most of them are benign and primary. In the pediatric age group, however, secondary or metastatic malignant tumors are more prevalent than are primary malignant tumors. Conceded that most cardiac tumors are benign histologically; still, if their location in the heart jeopardizes cardiac function, significant morbidity and mortality will ensue (1-4). For all the case reports of pediatric cardiac tumors from Iran, there is an apparent paucity of large collective works dedicated to this issue (5-7).

\section{Objectives}

The present study seeks to present and analyze the experience of our tertiary cardiac center (Rajaie Cardiovascular Medical and Research Center [RHC]) regarding pediatric cardiac tumors over an 8-year period. To the best of our knowledge, this 8-year review of 62975 pediatric patients referring for cardiac evaluation is one of the largest yet.

\section{Methods}

This retrospective observational study evaluated all patients aged under 16 years diagnosed with cardiac tumors in the Pediatric Cardiology Service of RHC between 2007 and 2015. Every patient's medical records were reviewed. The inclusion criteria consisted of patients younger than 16 years old, complete medical records, and at least two years follow up. The exclusion criteria included patients older than 16 years, incomplete medical records and less than two years follow up. Among 62975 pediatric in and out patients referred for cardiac evaluation, 41 patients were diagnosed with cardiac tumors. The patients' demographic characteristics including age and gender, as 
well as medical records on arrhythmias, incidental coexistent congenital heart lesions, tumor number and location, pathology (when available), and diagnostic imaging, were collected and tabulated. In patients with no pathological specimen, the tumor type was identified by comparing the tumor characteristics in echocardiography and other imaging modalities with descriptions in the literature (1-3). Two-dimensional transthoracic echocardiography was performed by pediatric cardiologists using GE Vivid 3 Ultrasound and Philips iE33 Ultrasound Machines. Transesophageal echocardiography was performed in the operating room and in patients with poor transthoracic windows. Based on echocardiographic criteria rhabdomyomas are well circumscribed, homogenous, commonly multiple and highly echogenic mass lesions that frequently regress over time. Fibromas are bright, well circumscribed, commonly single and may show areas of calcification. Myxomas are commonly single and located in left atrium. They are heterogenous, pedunculated, and mobile with irregular border. Teratomas are multiloculated and heterogeneous, due to cystic and calcified regions. When they are located within the pericardium, pericardial effusion is almost always present. Additionally, datas regarding surgery and its significant complications such as hemorrhage, infection, effusion, and cardiac dysfunction were marked. Follow-up data of between 2 and 8 years on tumor recurrence, mortality, and reoperation were also extracted, and each patient's outcome was collected. The confidentiality and anonymity of the information was considered by the researchers. The study protocol was approved by the Review Board of RHC. Prior to data collection, the license was received from the Ethics Committee of Iran University of Medical Sciences (IR.IUMS.FMD.REC.1398.068).

\subsection{Statistical Analysis}

Descriptive analyses and statistical comparisons were performed using the Student $\mathrm{t}, \chi^{2}, \mathrm{~K}^{2}$, and Fisher exact tests. A P value of less than 0.05 was considered statistically significant.

\section{Results}

\subsection{Patient Characteristics}

Cardiac neoplasms were diagnosed in 41 patients: 27 male and 14 female. The mean age of the study population was 4 years (range 2 days to 16 years).

\subsection{Tumor Characteristics}

Rhabdomyomas comprised the most common type (31.7\%), followed by fibromas (19.5\%) and myxomas (12.2\%).
The most common tumor location was the left ventricle, followed by the right ventricle, and both left and right ventricles, respectively. The other sites were the interventricular septum, the right atrium, the mitral valve, the left atrium, and the pericardium, in decreasing order. Figure 1 illustrates the tumor types with incidence of each type, and Figure 2 summarizes the descriptions of the patients and tumors.

Rhabdomyomas were diagnosed in 13 patients. One of these patients had a family history of tuberous sclerosis, and another one had skin lesions (café-au-lait spots). Multiple tumors $(>1)$ were present in 9 patients. Three patients with rhabdomyomas underwent surgery due to either an associated congenital heart disease (CHD) or the hemodynamic consequences of the tumor; none had significant complications after surgery. In 1 of these patients, the tumor caused severe mitral regurgitation. In all the patients with rhabdomyoma, the tumors regressed by time and even disappeared in some. Three patients were diagnosed with rhabdomyoma during fetal life; 1 of them experienced recurrent arrhythmias.

Fibroma was diagnosed in 8 patients: 4 girls and 4 boys. The tumor mainly involved the left ventricle and the interventricular septum. The only exception was a patient who selesion was on the aortic valve and entangled the ascending aorta; this patient was reported previously (5). The mass was removed surgically in 2 patients. Six of the patients with fibroma were followed conservatively by serial echocardiography. Three patients were placed on antiarrhythmic therapy and 2 patients had ablation as well. One patient was diagnosed with a fibroma during fetal life. A small atrial septal defect was incidentally found in one patient with a fibroma.

Myxoma was diagnosed in 5 patients: 4 boys and 1 girl. In 4 patients, the tumor was in the left atrium. In a 16-yearold boy, the tumor was in the right atrium; the boy also had the Ebstein anomaly of the tricuspid valve, which was repaired during surgery for tumor removal. The youngest patient with a myxoma was 10 months old. One patient experienced the embolic events of hemiparesis and speech loss. One patient with a myxoma had Wolff-Parkinson-White syndrome. All the patients with myxoma underwent total resection and subsequently suffered no recurrence. Undetermined tumor types were reported in 6 patients. In1 of these patients, the tumor was diagnosed by fetal echocardiography. Two of these patients did not continue the diagnostic process. One patient (a 4-day-old neonate) with an undetermined tumor type died very soon after the initial diagnosis; the patient had a huge tumor in the right ventricle and the intraventricular septum, causing left ventricular collapse, hypoplastic left heart syndrome-like symptoms, and respiratory distress. In 3 patients with undeter- 


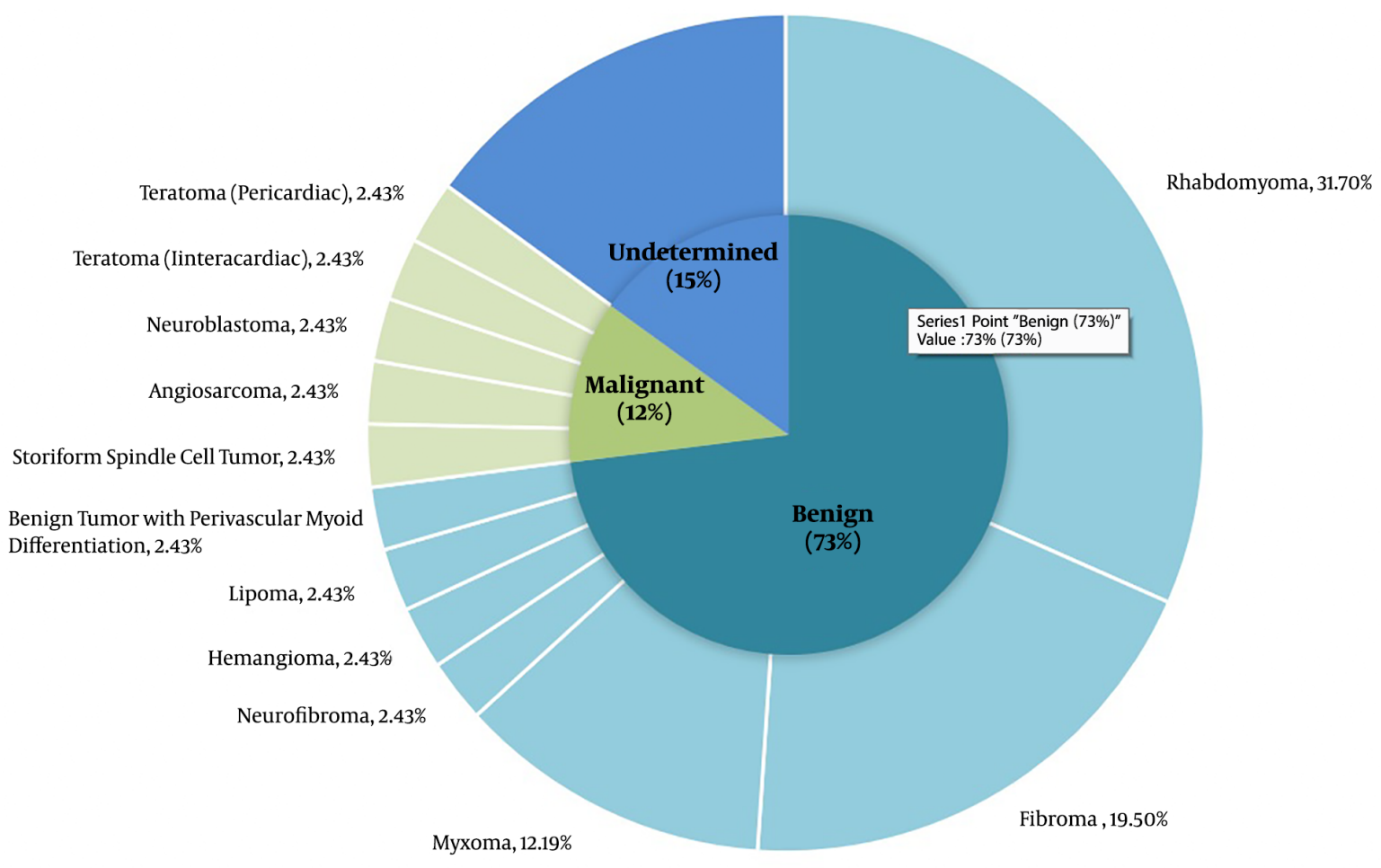

Figure 1. The pie chart depicts the types and incidence of the tumors in the study population.

mined tumor types, clinical or imaging findings were inadequate or controversial and there was no biopsy, rendering an accurate diagnosis impossible. The tumor size did not change in these 3 patients while they were followed via serial echocardiography. One of them received an implantable cardioverter-defibrillator after episodes of ventricular tachycardia.

Two patients were diagnosed with teratomas: intrapericardial and intracardiac. Both were classified in the malignant category because the tumor size increased during the follow-up. The intrapericardial teratoma was diagnosed during fetal life. The diagnosis in both patients was confirmed by magnetic resonance imaging (MRI), and both were on chemotherapy at the time of writing this article.

Among the patients with malignant tumors, 2 children died. An 11-year-old girl in our series suffered from an angiosarcoma; the tumor was at the right atrium-superior vena cava junction. Although the girl was placed on chemotherapy soon after the diagnosis was confirmed in her histological specimen, she expired. A 5-year-old girl was diagnosed with a metastatic neuroblastoma from the adrenal gland accessing the right atrium via the inferior vena cava; she underwent partial resection but died shortly afterward despite chemotherapy. A storiform spindle cell tumor in the right ventricle was diagnosed during the fetal life of a male neonate, who subsequently (at 35 days of life) uneventfully underwent the total resection of the tumor. The boy was in good condition at the time of writing this article.

Prenatal diagnosis was made in 7 patients by fetal echocardiography. Seven patients were also evaluated by computed tomography and 18 by MRI as supplemental imaging diagnostic modalities. Fourteen patients had histological specimens.

\section{Discussion}

Pediatric cardiac tumors are extremely rare; they are categorized into primary and secondary (or metastatic). Primary cardiac tumors are more common, and most of them are benign (about 10\% are malignant). Based on autopsy series, the prevalence of primary cardiac tumors in all age groups differ from 0.0017 to $0.28(8,9)$. The present retrospective study is the first and largest collective report from Iran regarding this rare disease. 


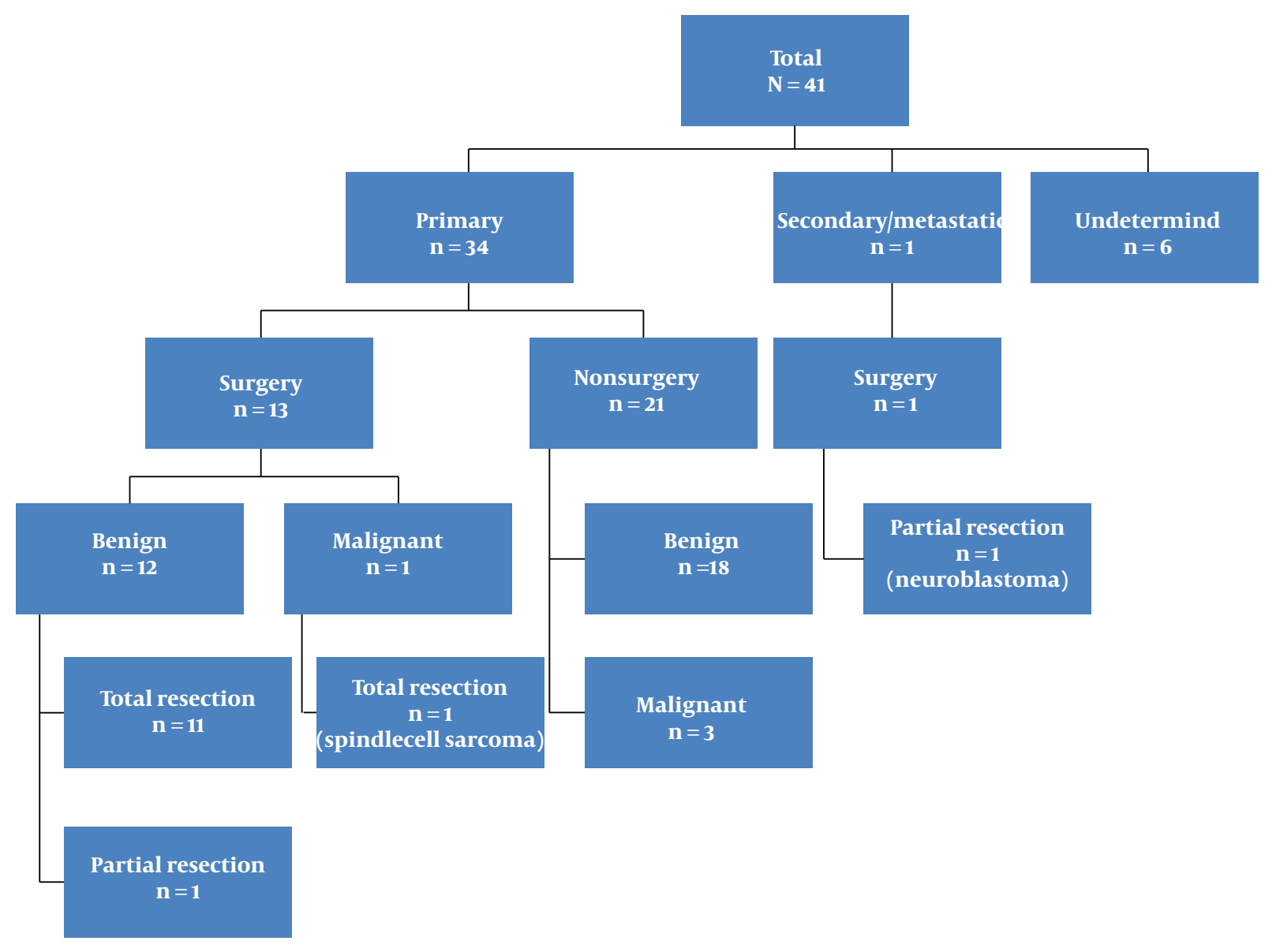

Figure 2. The flow chart presents a brief description of the tumors diagnosed in the study population.

The mean age of our patients was 4 years, which is almost similar to that reported in a study from China by Ying et al. (10) but different from reports from Portugal (23 mon) and Germany (1 mon) (11, 12). The disparity may be due to the delayed referral of our patients for cardiac evaluation.

In our study the prevalence of male patients with cardiac tumor was twice that of females. This finding was consistent with Shi et al. study on 166 pediatric with cardiac tumors (112 males, 54 females) (2). In another study this rate was $88 \%$ male gender for malignant cardiac tumor and $60 \%$ male for benign cardiac tumor (4).

Based on our study and analysis, primary cardiac tumors were more common than were secondary tumors (9.7\% vs 2.4\%), and most of the primary tumors were benign (73\%).

Rhabdomyoma was the most common tumor in our study. This finding is compatible with reports from other countries (9-13). In our study, the mean age of rhabdomy- omas was less than that of other tumors $(\mathrm{P}=0.001)$. According to the literature, rhabdomyoma typically appears before the first year of life and becomes smaller and regresses spontaneously with time (3). In all our patients, the tumor size decreased or totally disappeared with no recurrence. In our study rhabdomyoma was more common among boys $(\mathrm{P}=0.009)$. Three of our patients with rhabdomyoma underwent surgery; none of them had complications after surgery, with their serial echocardiographic follow-ups showing no relapse of the tumor and normal cardiac function. Rhabdomyoma is also the most common cardiac tumor diagnosed in fetal life and is highly associated with the tuberous sclerosis complex (8-15). Four of our patients were diagnosed with rhabdomyoma during fetal life and one of them had a family history of tuberous sclerosis and experienced arrhythmias as well.

Fibromas were the second most frequent tumors in our study,similar to the results of studies conducted by Paramés et al. (2009) (11), Stiller et al. (2001) (12), and 
Beghetti et al. (1997) (13). While a considerable number of investigations have considered fibromas to represent the second most common tumors in the pediatric population $(15,16)$, there are articles from the United States (4), China (10), and Australia (17), which have reported a high prevalence of myxomas by comparison with fibromas. Fibromas commonly present as single tumors, although they are multiple on rare occasions. In 1 of our patients, whose diagnosis was confirmed by biopsy as well, there were 2 fibroma masses. Stiller et al. (12) also reported 2 masses in 1 of their patients. Fibromas do not subside spontaneously but with the child's somatic growth, their size decreases in comparison with the heart size. Significant arrhythmias were more common in our series with fibromas than with other tumors $(3 / 8,37.5 \%)$. One of our patients with fibroma had nonsustained ventricular tachycardia and another one (No. 24) had atrioventricular reentrant tachycardia; both underwent ablation. In two other studies (2, 16) significant arrhythmias were more common in patients with fibromas. In an investigation by Holley et al. (8), a fibroma was found in 1 out of 19 pregnancies diagnosed with fetal cardiac tumors. Among our patients with fibromas, 1 case was diagnosed during fetal life.

Myxomas are the most common primary benign cardiac tumors in adults, whereas they are very rare in the pediatric population (1-3). In our study, myxomas comprised the third most common benign tumors. Also, in studies by Shi et al. (2017) (2) and Padalino et al. (2013) (18), myxomas were the third most common tumors. There are significant diversities among different studies. By way of example, none of the primary cardiac tumors was a myxoma in studies in Canada and Taiwan $(13,19)$. Positing that these differences might, to some extent, be due to the different ages of patients in different studies, Tzani et al. (20) argued that when young adult patients (age $<19 \mathrm{y}$ ) were included, myxomas would be the second most common tumor. Our youngest patient with a myxoma was 10 months old, and the other 4 were teenagers. None of our patients with myxomas was diagnosed during fetal life. In 2 studies, Elderkin and Radford (17) and Yuan (21) emphasized the rarity of fetal cardiac myxomas in the literature. Paladiniet al. (22) reported a 23-week-old fetus with a myxoma. Myxomas have a higher incidence in women. In contrast, in our study, boys outnumbered girls. Myxomas have the potential to cause embolic events either due to tumor debris or thrombus formation on the tumor, which can easily detach (1). In our series, 1 patient with a myxoma experienced a transient loss of speech and hemiparesis due to cerebrovascular accident. The most common location of myxomas is the left atrium (1-3, 9-15). In our series also, myxomas were more common in the left atrium. As myxomas have the potential of embolization, minimal manipulation and removal of the surrounding tissue during surgery should be considered (23-25). In our series, all the patients with myxomas underwent complete resection, and none had experienced recurrence by the time of writing this article.

Neurofibromas are benign tumors originating from the nerve sheath; they rarely involve the heart $(4,26)$. An 18-month-oldboy in our study had a neurofibroma and underwent surgery.

Although extremely rare in the pediatric population (1, $7,9,13$ ), a hemangioma, a lipoma, and a benign tumor with perivascular myoid differentiation were diagnosed in 3 of our patents.

Teratomas, albeit rare, constitute a major subgroup in pediatric cardiac tumors. They are germ cell tumors, containing all the 3 germ layers: endoderm, mesoderm, and ectoderm. Most commonly, teratomas are diagnosed under the age of 15 years, typically during the first year in neonates and even fetuses. They are considered to be primary and benign cardiac tumors, but they may undergo malignant degeneration $(1,3,12-15,27-29)$. Two of our patients had malignant teratomas: intrapericardial and intracardiac. The diagnosis was confirmed by MRI, and both were on chemotherapy at the time of writing this article. Patient No. 39 was diagnosed with a teratoma during fetal life.

Sarcomas account for the most frequent primary cardiac malignancy in both children and adults.

Spindle cell tumors represent another type of sarcomas, and they rarely involve the heart $(30,31)$. In our study, a 35-day-oldboy was diagnosed with this rare tumor during fetal life. In the reported cases, spindle cell tumors are located in the left atrium, pulmonary veins, great arteries, left ventricle, and pericardium. Our patient's tumor was in the right atrium. He underwent total resection and received adjuvant chemotherapy; he suffered no recurrence.

Metastatic cardiac tumors in the pediatric age group are not common in comparison with adults (14). Neuroblastomas constitute the third most common pediatric malignancy and can metastasize through the circulation and the lymphatic system; nevertheless, they rarely involve the heart (3). A metastatic neuroblastoma from the adrenal gland in our series accessed the right atrium via the inferior vena cava in a 5-year-old girl, who underwent partial resection but died shortly afterward despite chemotherapy. There are reports confirming the association between neuroblastomas and congenital heart disease (32). Our patient, however, had no associated congenital heart disease.

\subsection{Limitations}

The current investigation is a single-center study; a multicenter study with a larger sample volume will yield more robust data. The diagnosis of most of the tumors 
was, consequently, based on imaging modalities, especially echocardiography, rather than the histopathology of specimens, which is considered the gold standard.

\subsection{Conclusions}

Chiming in with previous reports, the majority of the cardiac tumors in a sample of Iranian children evaluated in the present study were primary and benign. Rhabdomyomas were the most common of all cardiac tumors diagnosed. Our findings also indicated that imaging modalities were unable to establish the histopathological diagnosis of a cardiac mass. Still, they conferred valuable anatomical and functional information, which was sufficient for a correct diagnosis.

\section{Acknowledgments}

We would like to express our deep gratitude to Mr. Farshad Amouzadeh and Mr. Ali Gord Jahanbeiglou for their kind cooperation.

\section{Footnotes}

Authors' Contribution: Maryam Moradian: conceptualization, methodology, investigation, writing - review \& editing. Batool Hejazi Zadeh: resources, software, data curation. Fariba Rashidi Ghader: visualization, supervision. Mohammadrafie Khorgami: conceptualization, methodology, software, data Curation, writing - original draft. Kambiz Mozaffari: project administration. Fatemeh Mohammad Sadeghi: supervision. Hamidreza Pouraliakbar: data curation.

Conflict of Interests: All authors of this study declare that they have no conflict of interest.

Ethical Approval: IR.IUMS.FMD.REC.1398.068.

Funding/Support: This study was not any funding.

\section{References}

1. Uzun O, Wilson DG, Vujanic GM, Parsons JM, De Giovanni JV. Cardiac tumours in children. Orphanet J Rare Dis. 2007;2:11. doi: 10.1186/17501172-2-11. [PubMed: 17331235]. [PubMed Central: PMC3225855].

2. Shi L, Wu L, Fang H, Han B, Yang J, Ma X, et al. Identification and clinical course of 166 pediatric cardiac tumors. Eur J Pediatr. 2017;176(2):25360. doi: 10.1007/s00431-016-2833-4. [PubMed: 28074279].

3. Allen HD, Shaddy RE, Penny DJ, Feltes TF, Cetta F. Cardiac tumors. In Marx GR, Moran AM, editors. Moss and Adams' heart disease in infants, children, and adolescents including the fetus and young adults. 9th ed. Lippincott Williams \& Wilkins (LWW); 2016.

4. Linnemeier L, Benneyworth BD, Turrentine M, Rodefeld $M$ Brown J. Pediatric cardiac tumors: a 45-year, single-institution review. World J Pediatr Congenit Heart Surg. 2015;6(2):215-9. doi: 10.1177/2150135114563938. [PubMed: 25870340].
5. Moradian M, Ziyaiefard M, Mozaffari. K. Atrial rhabdomyoma: a case report. Iran Heart J. 2014;15(2):39-42.

6. Aarabi Moghadam MY, Moradian M, Givtaj N, Mozaffari K. Intraluminal ascending aorta fibroma.J Teh Univ Heart Ctr. 2011;6(1):45-7.

7. Mozaffari K, Moradian M, Baharestani B, Mortezaeian H, Bakhshandeh $\mathrm{H}$. "Benign tumor with perivascular myoid differentiation", a rare yet interesting case report in a nine-month-old infant. Multidiscip Cardiovasc Ann. 2017;In Press(In Press). doi: 10.5812/mcardia.9366.

8. Holley DG, Martin GR, Brenner JI, Fyfe DA, Huhta JC, Kleinman CS, et al. Diagnosis and management of fetal cardiac tumors: a multicenter experience and review of published reports. J Am Coll Cardiol. 1995;26(2):516-20.

9. Ghadimi Mahani M, Lu JC, Rigsby CK, Krishnamurthy R, Dorfman AL, Agarwal PP. MRI of pediatric cardiac masses. AJR Am J Roentgenol. 2014;202(5):971-81. doi: 10.2214/AJR.13.10680. [PubMed: 24758649].

10. Ying L, Lin R, Gao Z, Qi J, Zhang Z, Gu W. Primary cardiac tumors in children: a center's experience. J Cardiothorac Surg. 2016;11(1):52. doi: 10.1186/s13019-016-0448-5. [PubMed: 27067427]. [PubMed Central: PMC4827228].

11. Paramés F, Freitas I, Martins JD, Trigo C, Pinto MF. Cardiac tumors: the 17-year experience of pediatric cardiology department. Rev Port Cardiol. 2009;28(9):929-40.

12. Stiller B. Primary cardiac tumours: when is surgery necessary? Eur J Cardiothorac Surg. 2001;20(5):1002-6. doi: 10.1016/s1010 7940(01)00951-4.

13. Mda MB, Gow RM, Haney I, Mawson J, Williams WG, Freedom RM. Pediatric primary benign cardiac tumors: A 15-year review. Am Heart J 1997;134(6):1107-14. doi: 10.1016/s0002-8703(97)70032-2.

14. Yuan SM. Fetal primary cardiac tumors during perinatal period. Pe diatr Neonatol. 2017;58(3):205-10. doi: 10.1016/j.pedneo.2016.07.004. [PubMed: 28043830].

15. Tao TY, Yahyavi-Firouz-Abadi N, Singh GK, Bhalla S. Pediatric cardiac tumors: clinical and imaging features. Radiographics. 2014;34(4):103146. doi: 10.1148/rg.344135163. [PubMed: 25019440].

16. Jayaprakash S. Clinical presentations, diagnosis, and management of arrhythmias associated with cardiac tumors. J Arrhythm. 2018;34(4):384-93. doi: 10.1002/joa3.12030. [PubMed: 30167009] [PubMed Central: PMC6111472].

17. Elderkin RA, Radford DJ. Primary cardiac tumours in a paediatric population. J Paediatr Child Health. 2002;38(2):173-7. doi: 10.1046/j.14401754.2002.00734.x. [PubMed: 12031001].

18. Padalino MA, Reffo E, Cerutti A, Favero V, Biffanti R, Vida V, et al Medical and surgical management of primary cardiac tumours in infants and children. Cardiol Young. 2014;24(2):268-74. doi: 10.1017/S104795111300022X. [PubMed: 23462142].

19. Wang JN, Yao CT, Chen JS, Yang YJ, Tsai YC, Wu JM. Cardiac tumors in infants and children. Acta Paediatr Taiwan. 2003;44(4):215-9. [PubMed: 14674225].

20. Tzani A, Doulamis IP, Mylonas KS, Avgerinos DV, Nasioudis D. Cardiac tumors in pediatric patients: A systematic review. World J Pediatr Congenit Heart Surg. 2017;8(5):624-32. doi: 10.1177/2150135117723904. [PubMed: 28901236].

21. Yuan SM, Yan SL, Wu N. Unusual aspects of cardiac myxoma. Anatol J Cardiol. 2017;17(3):241-7. doi: 10.14744/AnatolJCardiol.2017.7557. [PubMed: 28321109]. [PubMed Central: PMC5864986].

22. Paladini D, Tartaglione A, Vassallo M, Martinelli P. Prenatal ultrasonographic findings of a cardiac myxoma. Obstet Gynecol. 2003;102(5 Pt 2):1174-6. doi: 10.1016/s0029-7844(03)00079-6. [PubMed: 14607047].

23. Takach TJ, Reul GJ, Ott DA, Cooley DA. Primary cardiac tumors in in fants and children: immediate and long-term operative results. Ann Thorac Surg. 1996;62(2):559-64. [PubMed: 8694623].

24. Delmo Walter EM, Javier MF, Sander F, Hartmann B, Ekkernkamp A Hetzer R. Primary cardiac tumors in infants and children: Surgical strategy and long-term outcome. Ann Thorac Surg. 2016;102(6):20629. doi:10.1016/j.athoracsur.2016.04.057. [PubMed: 27344282]. 
25. Pathi VL, Royse A, Doig W, Pollock JC. Left atrial myxoma in a preschool child. Ann Thorac Surg. 1997;63(2):550-2. doi:10.1016/s00034975(96)00934-4. [PubMed: 9033343].

26. Friedman JM, Arbiser J, Epstein JA, Gutmann DH, Huot SJ, Lin AE, et al. Cardiovascular disease in neurofibromatosis 1 : report of the NF1 Cardiovascular Task Force. Genet Med. 2002;4(3):105-11. doi: 10.1097/00125817-200205000-00002. [PubMed:12180143].

27. Żalińska A, Korabiewska S, Krekora M, Michalak K, Kopala M, Cichos E, et al. Single fetal cardiac tumors and follow-up based on 13 cases from the fetal cardiac referral center in 1993-2017. Prenatal Cardiology. 2017;7(1):43-9. doi: 10.1515/pcard-2017-0007.

28. Burke A, Tavora F. The 2015 WHO classification of tumors of the heart and pericardium. J Thorac Oncol. 2016;11(4):441-52. doi: 10.1016/j.jtho.2015.11.009. [PubMed: 26725181].

29. Mirzaaghayan MR, Shabanian R. A huge intrapericardial teratoma in an infant. Pediatr Cardiol. 2008;29(6):1122-3. doi: 10.1007/s00246-0089264-6. [PubMed: 18600368].

30. Muturi A, Kotecha V, Ruturi J, Muhinga M, Waweru W. High-grade spindle cell sarcoma of the heart: a case report and review of literature. J Cardiothorac Surg. 2015;10:46. doi: 10.1186/s13019-015-0245-6. [PubMed: 25888133]. [PubMed Central: PMC4382851].

31. White RM, Sheppard RC, Wassmer PC, Wilson CH, Morales CA. Left atrial spindle cell sarcoma in a 34-year-old woman. CASE (Phila). 2018;2(4):151-5. doi: 10.1016/j.case.2018.03.001. [PubMed: 30140782]. [PubMed Central: PMC6104146].

32. van Engelen K, Merks JH, Lam J, Kremer LC, Backes M, Baars MJ, et al. Prevalence of congenital heart defects in neuroblastoma patients: a cohort study and systematic review of literature. Eur J Pediatr. 2009;168(9):1081-90. doi: 10.1007/s00431-008-0891-y. [PubMed: 19089449]. [PubMed Central: PMC2714905]. 